\title{
Toxicogenomic applications of Chinese rare minnow (Gobiocypris rarus) in aquatic toxicology is
}

\author{
Xuefang Liang ${ }^{\mathrm{a}}$, Jinmiao Zha ${ }^{\mathrm{b}, *}$ \\ a College of Environment and Resources, Inner Mongolia University, Hohhot 010021,China \\ ${ }^{\mathrm{b}}$ Key Laboratory of Drinking Water Science and Technology, Research Center for Eco-Environmental Sciences, Chinese Academy of Sciences, Beijing 100085, China
}

\section{A R T I C L E I N F O}

\section{Article history:}

Received 15 September 2015

Received in revised form 9 June 2016

Accepted 15 June 2016

Available online 21 June 2016

\section{Keywords:}

Rare minnow (Gobiocypris rarus)

Toxicogenomics

Microarray

Proteomics

Endocrine disrupting chemical

\begin{abstract}
A B S T R A C T
Rare minnow (Gobiocypris rarus), a Chinese native species, are an excellent emerging model organism for aquatic toxicity testing and chemical safety assessment. "Big data" omics approaches (i.e., genomics, proteomics, and metabolomics) to inform mechanistic toxicology are now applied to studies in rare minnows to better understand toxicity and molecular pathways perturbed by chemicals. This review highlights recent applications of toxicogenomics to study changes in the gene and protein expression profiles in rare minnows in response to chemicals. Here we briefly describe studies that utilized cDNA microarrays in characterization of the cellular effects of rare minnows in single and mixed chemical exposures. Then we compare gel-based proteomics studies in liver of rare minnows following treatment with endocrine disrupting chemicals including $17 \beta$-estradiol, $17 \alpha$-methyltestosterone, pentachlorophenol, and perfluorooctanoic acid. A total of 90 proteins identified in these studies were functionally annotated and categorized. These responsive proteins have roles in biological processes that include metabolism (37.8\%), response to oxidation/chemicals (16.7\%), signal transduction (11.1\%), transport (10\%), cytoskeleton (6.7\%) and others (17.8\%). In addition, recent investigations of endocrine disrupting effects and neurotoxicity of benzotriazole, an emerging contaminant, are summarized. The objective is to continue to enrich genome and protein databases for this species and to integrate molecular datasets to consider temporal effects and complex regulation at the level of the genome and proteome.
\end{abstract}

(C) 2016 Elsevier Inc. All rights reserved.

\section{The Chinese rare minnow (Gobiocypris rarus) as an emerging model in aquatic toxicology}

Fish species comprise a major percentage within the vertebrate group, with a distribution that covers practically all aquatic ecosystems (Forné et al., 2010). Teleosts represent more than half of all vertebrate species and are adapted to a wide range of marine and freshwater habitats (Nelson, 1994). As many species are the top in aquatic food chains, fish may directly affect the health of humans via consumption as a chemical sink. Thus, fish are often used in in vivo tests, which are based on definitive indicators and show a direct cause-and-effect relationship for endpoints of ecological concern (Hontela, 1998). Zebrafish (Danio rerio), fathead minnow (Pimephales promelas), rainbow trout (Oncorhynchus mykiss), and medaka (Oryzias latipes) have been recommended as aquatic test species by the International Organization

\footnotetext{
it This paper is a contribution to the Special Issue on Environmental Omics and Toxicology.

* Corresponding author at: Key Laboratory of Drinking Water Science and Technology, Research Center for Eco-Environmental Sciences, Chinese Academy of Sciences, 18 Shuangqing Road, Haidian District, Beijing 100085, China.

E-mail address: jmzha@rcees.ac.cn (J. Zha).
}

for Standardization (ISO). However, to date, few in vivo screening tests that are relevant to the exposure of Chinese native fish to chemicals have been identified and validated. Since many potential pollutants are likely to be released directly into ecosystem in China, it is necessary to develop in vivo screening tests for chemical safety assessment using Chinese native species (Qun-Fang et al., 2002).

Rare minnow (Gobiocypris rarus) is a fish species of the largest family, the Cyprinidae. The fish is mostly distributed in upstream waters in the Yangtze River, Sichuan Province, China. They are small in size (30-80 $\mathrm{mm}$ in total length), adaptable to a wide temperature range $\left(0-35^{\circ} \mathrm{C}\right)$, and easy to culture in the laboratory (Wang and Cao, 1997; Zhou et al., 1995). They have a relatively short life cycle, and spawn hundreds of eggs with high fertilization and hatching rates (Wang, 1992; Zha et al., 2008). In the aquarium, spawning may occur throughout the year under controlled conditions (temperature, food, etc.), though the breeding season in the field lasts from March to November, when the water temperature is above $14{ }^{\circ} \mathrm{C}$ (Wang, 1992; Wang and Cao, 1997). Rare minnow shows continuous batch spawning, and the average number of eggs spawned by individual females is estimated to be 266 in the laboratory. Intervals between spawning sessions range from 2 to 9 days, with a mean of 4.5 days (Wang, 1992; Wang and Cao, 1997). The developmental period of rare minnow from 
fertilization to hatching is very brief $\left(72 \mathrm{~h}\right.$ at $\left.26^{\circ} \mathrm{C}\right)$. Moreover, the duration of embryonic development is prolonged largely by low temperature and accelerated by high temperature. For example, the embryonic developmental period lasts $312 \mathrm{~h}$ at $13{ }^{\circ} \mathrm{C}$ while it takes only $48 \mathrm{~h}$ at $31.6{ }^{\circ} \mathrm{C}$ (Chang et al., 1995). Compared with zebrafish, whose duration of embryonic development is approximately $96 \mathrm{~h}$ at $26^{\circ} \mathrm{C}$, rare minnow may be more advantageous for embryological studies if shorter time periods are desired (Chang et al., 1995). At water temperatures ranging from 24.7 to $31.8{ }^{\circ} \mathrm{C}$, the postembryonic development of rare minnow lasts for 25 to 30 days, and the larvae grow uniformly with a growth rate of $0.52 \mathrm{~mm}$ per day (Wang et al., 1998). Fish attain sexual maturity in 4 months after hatch, and males and females are easily distinguished as adults according to secondary sexual characteristics (Wang, 1992).

These characteristics mentioned above make the rare minnow an excellent model for aquatic toxicity testing. Numerous studies have selected rare minnow as an in vivo model to study the effects of endocrine disruptors (e.g. diethylstilbestrol) and of hepatotoxic contaminants (e.g. perfluorooctanoic acid) implicated in the incidence of liver tumors in mammals (Wei et al., 2008b; Zha et al., 2007, 2008; Zhang et al., 2008a; Zhong et al., 2005). In the year of 2014, a national standard named 'Chemicals-rare minnow (Gobiocypris rarus) acute toxicity test' (GB/T 29763-2013) was promulgated in China (General Administration of Quality Supervision Inspection and Quarantine of the People's Republic of China and Standardization Administration of the People's Republic of China, 2014). Thus, the rare minnow, as an emerging model in aquatic toxicology, is expected to be more widely used in chemical safety assessment and environmental toxicological studies in the future.

In this review, we will focus on the development of toxicogenomics and its recent applications in toxicity assessment and toxicological pathway analysis for endocrine disrupting chemicals (EDCs), highlighting some examples using studies conducted with emerging pollutants in rare minnows.

\section{Toxicogenomics in aquatic toxicology studies}

Toxicogenomics is the study of how genomes respond to environmental stressors or toxicants. Specifically, it combines omics technologies (i.e., genomics, proteomics, and metabolomics) and bioinformatics with toxicology to understand the role of gene-environment interactions in disease and dysfunction (Waters and Fostel, 2004). The three principal goals of toxicogenomics have been proposed to be (1) understanding the relationship between environmental stress and human disease susceptibility; (2) identifying useful biomarkers of disease and exposure to toxic substances; (3) and elucidating the molecular mechanisms of toxicity (Gatzidou et al., 2007). With the development of highthroughput biological technologies, toxicogenomics has evolved from early gene-expression studies, toward more mature investigations that integrate several-omics domains with toxicology and pathology data (Schmidt, 2002).

Toxicogenomics was initiated after the development of the first highdensity microarrays, or sets of miniaturized binding areas that were used to measure DNA fragments, antibodies, or proteins (Nuwaysir et al., 1999). Gene expression is a sensitive indicator of toxicant exposure, disease state, and cellular metabolism and thus represents a unique way of characterizing how cells and organisms adapt to changes in the external environment (Lettieri, 2006; Mitra et al., 2012). Genomic information of an organism can be used to design microarrays to determine which genes are up- or down-regulated in response to an environmental perturbation, such as exposure to a toxic chemical. The global detection and analysis of gene expression in this fashion are termed "transcriptomics" (Afshari et al., 1999; Brown and Botstein, 1999; Duggan et al., 1999; Ankley et al., 2006). Transcriptomics can both provide information about the mechanism of action of toxicants and can result in a "genetic signature" which allows for more rapid screening of unknown or suspected toxicants on the basis of their similarity to known toxicants
(Hawliczek et al., 2012; Kosmehl et al., 2012). The field of toxicogenomics has progressed rapidly since the application of DNA chips to toxicology was proposed in the late 1990s (Afshari et al., 1999). In aquatic toxicology, microarrays were rapidly developed for aquatic species and have been widely applied to aquatic toxicogenomics since the earliest application in catfish for investigating genes regulated by cold acclimation (Ju et al., 2002, 2007; Larkin et al., 2002a, 2002b).

In the transcription step that initiates the conversion of genetic information into proteins, not all mRNA sequences are transcribed from DNA. In addition, many proteins are modified (e.g., phosphorylation, posttranslational cleavage) before becoming physiologically active. Consequently, proteomics-the global evaluation of protein profiles-provides additional critical insights into biological pathways (Garcia-Reyero and Perkins, 2011; Martyniuk and Denslow, 2009). With the potential of achieving high-throughput analysis of effects on protein populations and sub-populations, proteomics allows researchers to better understand toxic modes of action and to identify novel ecotoxicological biomarkers (Dowling and Sheehan, 2006; Nesatyy and Suter, 2007). As with transcriptomics, a rapid evolution has occurred with proteomic methods capable of providing broad characterization of the proteins expressed within cells, organs, or whole organisms (Ahmad and Lamond, 2014; Nesatyy and Suter, 2007). A proteomic strategy typically includes protein isolation, proteolytic digestion and peptide fractionation (for bottom-up and middle-down approaches), and separation steps. This is followed by mass spectrometry (MS) analyses to identify specific proteins (Zhang et al., 2013). After the first application in aquatic toxicology by Shepard and Bradley (2000) and Shepard et al. (2000) who analyzed the proteomic response of mussels (Mytilus edulis) exposed to polychlorinated biphenyls, copper, and salinity stress, proteomic techniques have been widely applied to fishes as well as other aquatic invertebrates (Sanchez et al., 2011).

Metabolomics is the field of science that characterizes endogenous and exogenous low molecular mass metabolites within a cell, tissue, or bio-fluid of an organism in response to external stressors such as disease, contaminant exposure, or nutritional imbalances (Lankadurai et al., 2013). Metabolomics use analytical techniques such as nuclear magnetic resonance (NMR) spectroscopy or mass spectrometry to identify metabolites in pathways or classes of compounds (Viant and Sommer, 2013). The capacity to detect subtle molecular changes, and the comprehensive nature of metabolite measurements, have resulted in the increased popularity of metabolomics in aquatic toxicology (Lankadurai et al., 2013; Tuffnail et al., 2009). Because of the potential of detecting biomarkers of stress and delineate the modes of action of xenobiotics, this technique has been applied to experimental studies of selective exposure in aquatic organisms in both invertebrates and fish (Li et al., 2014; Zhang et al., 2011). However, to our knowledge, there have been no metabolomic applications reported in rare minnows as of yet. Hence in this review, we only discuss the applications of microarrays and proteomics in rare minnows (Fig. 1).

\section{Applications of toxicogenomics based on microarrays in rare minnows}

DNA microarray technology has been applied to the analyses of gene expression profiling in response to chemical exposure and to identification of chemical effects to rare minnow. Wei et al. (2008b) used a custom cDNA microarray containing 1773 unique genes to investigate the hepatic gene expression profile in rare minnows treated with $10 \mathrm{mg} / \mathrm{L}$ perfluorooctanoic acid (PFOA) for 28 days. They found 124 and 171 genes significantly altered in males and females, respectively, of which 43 genes were commonly regulated in both sexes. According to the biological process of these genes, the authors speculated that PFOA could suppress fatty acid biosynthesis and transport and exert endocrine disruption effect in rare minnows. Combined with other omics technologies (e.g. proteomics), toxicogenomics based on microarray analysis may further improve our understanding of adverse effects 


\section{Chemical exposure in rare minnows}

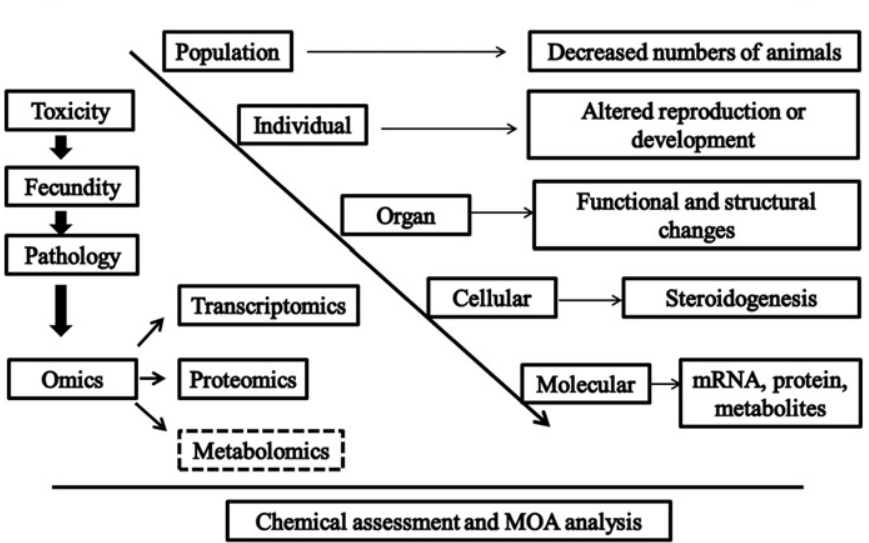

Fig. 1. Schematic representation of an approach to integrate toxicogenomic data for chemical assessment and MOA analysis in rare minnow. Connecting steps across biological levels of organization for effects of chemicals in rare minnow are reflected by arrows. Toxicogenomic data can be analyzed for differentially expressed genes, proteins, and metabolites. Consecutively, these data can be grouped into affected pathways. Jointly with toxicity data, toxicogenomic data evaluation can inform mechanisms of action for a chemical (e.g. PFOA). Dotted box indicates that application of metabolomics in rare minnow is not discussed in this review.

and molecular pathways regulated in fish tissues by chemical exposure (Calzolai et al., 2007; Martyniuk and Denslow, 2012). For example, in a study of genomic and proteomic response to dieldrin, a neurotoxic and endocrine disrupting chemical, sub-chronic dietary exposures were conducted in largemouth bass. Microarrays, proteomics and pathway analysis demonstrates that dieldrin alters the abundance of mRNAs and proteins in the hypothalamus that are associated with cell metabolism, cell stability and integrity, stress, and DNA repair (Martyniuk et al., 2010b).

In addition, toxicogenomics provides a tool to improve our understanding and predictability of combined effects provoked in organisms through exposure to mixtures of chemicals. It allows observing the interplay between impacted environmental conditions and dynamic responses of organisms at the genetic level (Altenburger et al., 2012). This approach has been applied in the characterization of the cellular effects of rare minnows in single and mixed chemical exposures. In a previous study, effects caused by six individual polyfluorinated and perfluorinated compounds (PFCs) and their mixtures in hepatocytes of rare minnows were detected using a cDNA microarray (Wei et al., 2009). In the above study, the discrepancy of gene expression profiles between single and mixed PFCs exposures was illustrated; a particular gene set (e.g. calmodulin,intelectin,sox9b, etc.) was consistently regulated by both groups, whereas a number of genes affected by mixtures were not altered relative to any of the individual components (e.g. ovary-specific C1q-like factor, uncoupling protein 1, etc.). These unique altered genes provide clues as to the molecular modes of action of PFC in co-exposures and may allow for the validation of a set of PFCresponsive genes in order to monitor and assess toxicological risks of PFC exposure in different environments.

\section{Applications of toxicoproteomics in rare minnow for endocrine disrupting chemicals}

Rare minnows have been most applied as a model for investigating endocrine disrupting chemicals (EDCs). Rare minnows are an excellent test fish for EDCs toxicology studies and have been used in both at specific life stages or multi-generation exposures (Zha et al., 2007, 2008; Zhong et al., 2005). Initially, physiological endpoints of the fish including secondary sexual characteristics, gonadosomatic indices, plasma steroids, and gonadal histology have been used for EDCs assessment
(Zha et al., 2007, 2008). These endpoints achieved good sensitivity and efficiency. For example, Zha et al. (2008) assessed mortality, deformities, reproductive parameters, plasma vitellogenin, and histopathology, and demonstrated that $17 \alpha$-ethinylestradiol (EE2) affects reproduction of rare minnow as low as $0.2 \mathrm{ng} / \mathrm{L}$ concentration in a multi-generation exposure.

Furthermore, transcriptomic analysis improved our understanding of molecular pathways regulated in fish tissues by EDCs exposure and strengthened linkages across biological levels of organization. Genes related to hypothalamic-pituitary-gonadal (HPG), hypothalamicpituitary-thyroidal (HPT) and hypothalamic-pituitary-adrenal (HPA) axes were cloned and quantified to detect adverse outcomes caused by EDCs, such as vitellogenin (VTG), estrogen receptor (ER), thyroid hormone receptor alpha (tr $\alpha$ ), deiodinase (dio1 and dio2), glucocorticoid receptor (gr) (Li et al., 2009a, 2009b; Yang et al., 2010a, 2010b; Zhang et al., 2008b, 2008c). For example, Li et al. (2009b) cloned thyroid hormone-related genes (such as tr $\alpha, \mathrm{d} 1$ and $\mathrm{d} 2$ ) and demonstrated that 3-amino-1,2,4-triazole and acetochlor have thyroid disruption effects on these genes in rare minnow.

Functional genomics also includes studying how genomic changes correlate to changes at the protein level. Proteomic analyses provide global protein information including protein variants and posttranslational modifications, and the characterization of protein-protein interactions (Wetmore and Merrick, 2004). Quantitative proteomic approaches include gel-based (e.g., two dimensional electrophoresis; 2$\mathrm{DE}$, differential in gel electrophoresis; DIGE) and gel-free methods that can be separated further into labeling approaches such as stable isotope labeling (SILAC), isotope coded affinity tags (ICAT), and isobaric tagging (iTRAQ) and label-free approaches (e.g., spectral counting and absolute quantitation) (Baggerman et al., 2005; Patel et al., 2009). In aquatic toxicology, DIGE and iTRAQ are the most widely used technologies to identify and characterize protein bioindicators of adverse effect (Martyniuk et al., 2012a). Up to now, several toxicological studies based on 2-DE and DIGE approaches have been carried out in rare minnows (Fang et al., 2010, 2014; Liang et al., 2014a; Wei et al., 2008a) (Table 1). Liang et al. (2014a) compared the response of the liver proteome of male rare minnows treated with $17 \beta$-estradiol (E2) and females treated with $17 \alpha$-methyltestosterone (MT). Among 47 differentially expressed proteins, pyruvate carboxylase was the only common altered protein in both groups. This protein was validated as a potential biomarker for exogenous steroid chemicals in the liver of fish. Efforts to better understand the impact of EDCs on the proteome of rare minnow also include studies using pentachlorophenol (PCP) (Fang et al., 2010), PFOA in liver (Wei et al., 2008a) and PCP and 2,4,6-trichlorophenol (TCP) in ovary (Fang et al., 2014).

To characterize the fish liver proteome in response to EDCs, all 90 proteins identified in previous studies in the rare minnows exposed to 17 $\beta$-estradiol (E2), 17 $\alpha$-methyltestosterone (MT) (Liang et al., 2014a), PCP (Fang et al., 2010) and PFOA (Wei et al., 2008a) were functionally annotated by searching Gene Ontology (http://www.geneontology. org/). The biological functions most represented by proteins detected in the liver of rare minnows exposed to EDCs included metabolism (37.8\%), response to oxidation/chemicals (16.7\%) and signal transduction (11.1\%) (Fig. 2). Among these proteins, 17.8\% proteins were related to other functions which were not classified to a group (Fig. 2). These proteins may be important in some critical pathways but the information about them is limited. Several proteins associated with chemical or stress responses were identified in these studies (e.g. vitellogenin 1 precursor, eukaryotic translation elongation factor 2, glutathione peroxidase 1, natural killer cell enhancement factor), which provide important clues for toxic mechanisms of action of EDCs. What's more, modes of action may be different among different EDCs and combined exposures may exacerbate the toxicity. For example, in PFOA exposure, fatty acid metabolism and oxidative stress are the primary biological processes impacted, however, in PCP exposure, ATP transport and protein metabolism appear to be an important part of the toxic effects. 
Table 1

Summary of proteomic studies in aquatic toxicological research with rare minnows.

\begin{tabular}{|c|c|c|c|c|c|c|}
\hline Tissue & $\begin{array}{l}\text { Chemical } \\
\text { treatment }\end{array}$ & $\begin{array}{l}\text { Exposure } \\
\text { period }\end{array}$ & Methods $^{\mathrm{a}}$ & $\begin{array}{l}\text { Proteins } \\
\text { identified }^{\text {b }}\end{array}$ & Proteins of interest ${ }^{\mathrm{c}}$ & References \\
\hline Liver & PFOA & $28 \mathrm{~d}$ & 2-DE-MALDI-TOF-MS & $15(\mathrm{M}) ; 10(\mathrm{~F})$ & $\begin{array}{l}\uparrow \text { Muscle fatty acid binding protein (M) } \\
\uparrow \text { Peroxiredoxin (M) } \\
\uparrow \text { Translationally controlled tumor protein (F) } \\
\uparrow \text { Methionine sulfoxide reductase B (F) } \\
\downarrow \text { Phenylalanine hydroxylase (F) }\end{array}$ & Wei et al. (2008a) \\
\hline Liver & PCP & $28 \mathrm{~d}$ & 2-DE-MALDI-TOF-MS & $6(\mathrm{M}) ; 12(\mathrm{~F})$ & $\begin{array}{l}\downarrow \text { Liver-basic fatty acid-binding protein (F) } \\
\downarrow \text { Mitochondrial ATP synthase (F) } \\
\uparrow \text { Glutathione peroxidase (M) } \\
\uparrow \text { Fructose-1,6-bisphosphatase (M) }\end{array}$ & Fang et al. (2010) \\
\hline Ovary & PCP, TCP & $28 \mathrm{~d}$ & 2-DE-MALDI-TOF-MS & 14 & $\begin{array}{l}\uparrow \text { Vitellogenin precursor } \\
\uparrow \text { Small ubiquitin-related modifier } \\
\uparrow \text { Galectin family member } \\
\downarrow \text { L-isoaspartate O-methyltransferase }\end{array}$ & Fang et al. (2014) \\
\hline Liver & E2, MT & $120 \mathrm{~d}$ & DIGE-MALDI-TOF-MS & 23 (E2); 24 (MT) & $\begin{array}{l}\uparrow \text { Pyruvate carboxylase, mitochondrial (E2) } \\
\downarrow \text { Vitellogenin } 1 \text { precursor (MT) } \\
\downarrow \text { Eukaryotic translation elongation factor } 2 \text {, like (MT) } \\
\downarrow \text { Pyruvate carboxylase, mitochondrial (MT) }\end{array}$ & Liang et al. (2014a) \\
\hline Brain & BTR & $28 \mathrm{~d}$ & 2-DE-MALDI-TOF-MS & 41 & $\begin{array}{l}\uparrow \text { Hippocalcin } \\
\uparrow \text { Creatine kinase, brain b } \\
\uparrow \text { Glutamine synthetase } 1 \\
\uparrow \text { Flotillin } 1 \text { a } \\
\downarrow \text { Mitogen-activated protein kinase } 1\end{array}$ & Liang et al. (unpublished data) \\
\hline Liver & BTR & $28 \mathrm{~d}$ & DIGE-MALDI-TOF-MS & 46 & $\begin{array}{l}\uparrow \text { Hydroxysteroid dehydrogenase-like protein } 2 \\
\uparrow \text { Aldehyde dehydrogenase } 2 \\
\downarrow \text { Sulfotransferase family } 1 \text {, cytosolic sulfotransferase } 6 \\
\downarrow \text { Apolipoprotein A-I }\end{array}$ & Liang et al. (unpublished data) \\
\hline
\end{tabular}

For fish toxicogenomic studies, exploring gene-protein relationships is also a critical part to provide more insightful information in predicting toxic pathways of chemicals over a given time period. However, the relationship between transcription of mRNA and the abundance of protein is not always direct (Martyniuk et al., 2012b). In the liver of rare minnow after treatment with E2, MT (Liang et al., 2014a), PCP (Fang et al., 2010) and PFOA (Wei et al., 2008a), researchers demonstrated that gene-protein correspondence from data collected with real-time PCR data and 2D gel electrophoresis was highly correlated for some genes and proteins (e.g. fructose-1,6-bisphosphatase; F-1,6-BP, liver fatty acid binding protein; L-FABP, pyruvate carboxylase; PC) but not for others (e.g. glutamate dehydrogenase; GDH) (Fig. 3). These inconsistent results in directional change between mRNA and protein are likely due to the regulation (synthesis and breakdown) of both mRNAs and proteins which have different kinetics and turnover rates. In the liver of female rare minnow after treatment with MT, the abundance of

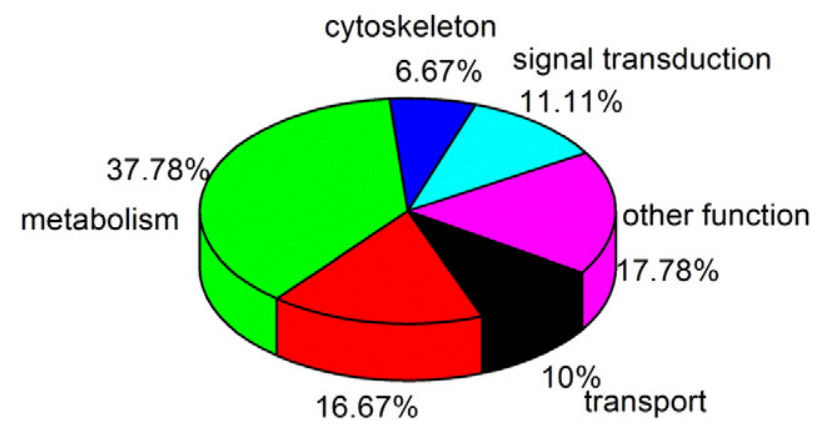

response to stress/chemicals

Fig. 2. The biological function categories for proteins detected in the liver proteome of rare minnows. A total of 90 altered proteins which were identified in livers of rare minnows exposed to E2, MT, PCP and PFOA were functionally annotated and shown in the pie chart (data from Fang et al., 2010; Liang et al., 2014a; Wei et al., 2008a).
VTG1 protein showed the trend of down-regulation in all MT-treated groups while the mRNA level of vtg1 was up-regulated in 1 and $10 \mathrm{ng} / \mathrm{L}$ MT groups (Liang et al., 2014a). Similarity, in a previous study, Martyniuk et al. (2010a) observed that microtubule associated protein tau (mapt) mRNA levels were decreased while MAPT protein abundance was increased in telencephalon of male fathead minnows after $17 \alpha$-ethinylestradiol exposure.

Other regulatory pathways related to transcription and translation, such as alternative splicing of mRNA, and post-translational modification of proteins will also contribute significantly to gene-protein relationships. In the liver of rare minnows after treatment with MT, a completely opposite expression patterns of alcohol dehydrogenase $(\mathrm{ADH})$ protein and its mRNA levels were observed which may be attributed to such complex regulatory mechanisms (Fig. 3). In several studies that combined microarray and proteomic data, the transcripts and proteins used in the analysis also showed significant changes in steady state levels (de Sousa Abreu et al., 2009; Martyniuk et al., 2012b; Popesku et al., 2010). For example, de Sousa Abreu et al. (2009) demonstrated that squared Pearson's correlation coefficients for gene-protein studies range from 0.20 to 0.47 in bacteria, $0.34-0.87$ in yeast, and $0.09-0.46$ in multi-cellular organisms. Apparently, the regulatory pathways of different molecules are highly specific and diverse and can result in poor gene-protein relationships.

\section{Toxicoproteomic applications in rare minnows for toxicological discovery of emerging contaminants}

In recent years, new classes of aquatic pollutants have received attention due to their introduction into the environment, unforeseen effects associated with the pollutants, or enhanced analytical techniques presently capable of detecting them. Many of these emerging contaminants are not well-documented in their toxicity, making predictions regarding their environmental fate or risk to aquatic organisms difficult (Poynton and Vulpe, 2009). Since the development of high-throughput toxicogenomics has effectively driven the screening program that 
A
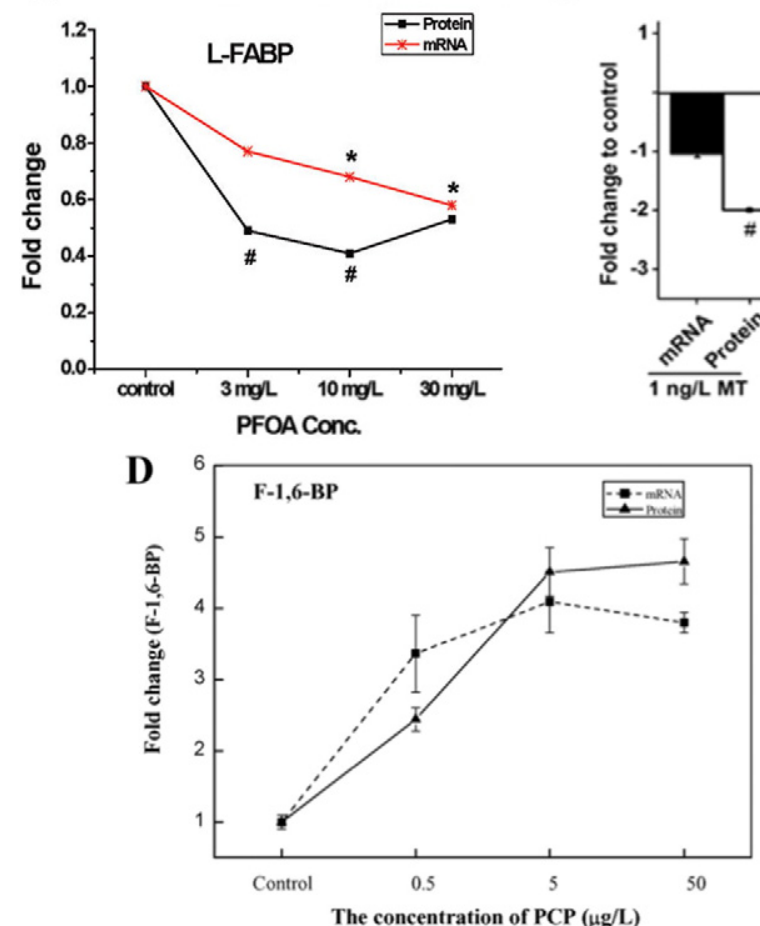

B

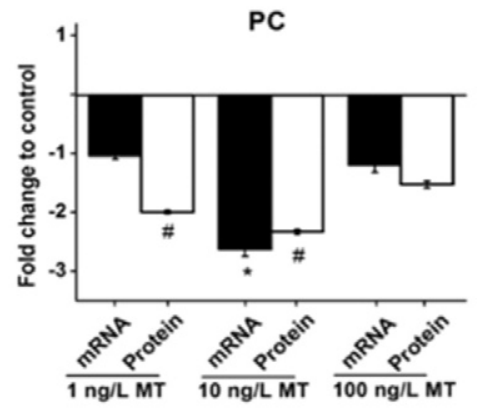

C

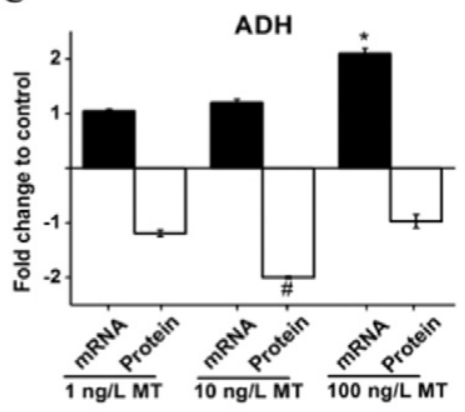

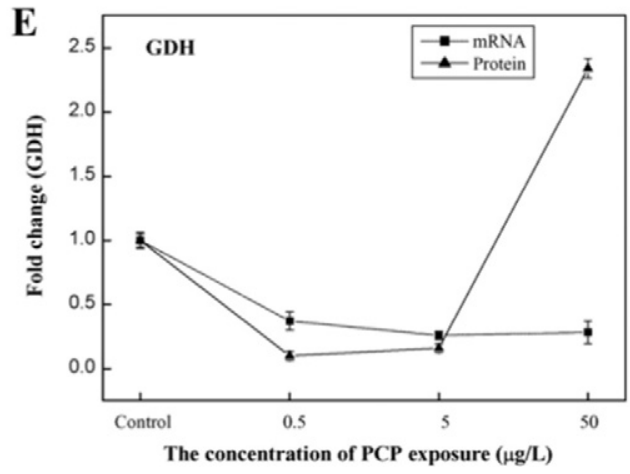

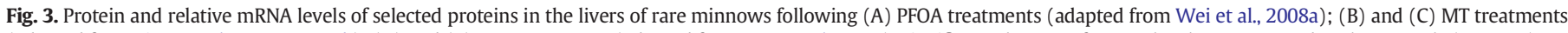

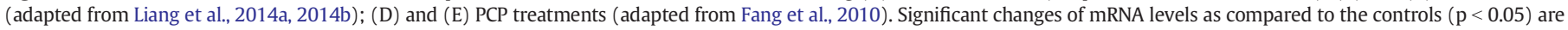
indicated by asterisks $\left({ }^{*}\right)$, and pound sign (\#) refers to an average increase or decrease higher than 2-fold in protein levels compared to the controls.

warrant further development and testing, complementary toxicogenomic data facilitate the monitoring and management of these chemicals (Boverhof and Zacharewski, 2006). Moreover, toxicogenomic data have the potential to inform many aspects of risk assessment including hazard identification and characterization, mechanisms of action, inter- and intraspecies differences in modes of action, exposure assessment, and dose-response assessment. It is therefore likely that a better understanding of molecular changes will enable greater insight into the factors behind the observed variability in susceptibility to chemical exposure that can be seen in aquatic organisms (Miracle and Ankley, 2005; Oberemm et al., 2005).

Our group recently leveraged proteomic methods to evaluate the toxicity and predict mechanisms of action of emerging contaminants, with the objective of integrating proteomic data in chemical risk assessment. We have used the gel-based proteomic method successfully in the rare minnow to investigate the endocrine disrupting effect and neurotoxicology of benzotriazole (BTR), an emerging pollutant which has been widely detected in aquatic environments (Liang et al., unpublished data). Reports on endocrine disrupting effects and toxic mechanisms of BTR to aquatic organisms are limited. To investigate its toxicity, we investigated the pathology, steroid hormone levels and the transcriptional profile of the HPG axis in rare minnow. The results indicate that BT has endocrine disrupting effects and could adversely affect the rare minnows in a sex-dependent manner (Liang et al., 2014b). Based on this study, DIGE was applied to observe the changes in protein abundance in the liver of rare minnow (Liang et al., unpublished data). In this experiment, 46 altered proteins were identified, of which 27 proteins were up-regulated and 16 were down-regulated. Among them, 3 proteins involved in sterol metabolic process (including hydroxysteroid dehydrogenase-like protein 2 , sulfotransferase family 1 , and cytosolic sulfotransferase 6, apolipoprotein A-I) were identified. Additionally, 4aminobutyrate aminotransferase, mitochondrial, which is associated with gamma - aminobutyric acid (GABA) metabolic process, was found to be increased by 1.52 fold after $0.05 \mathrm{mg} / \mathrm{L}$ BT treatment. GABA is an important neurotransmitter and plays critical role in neuroendocrine. GABA receptor is demonstrated as the target of some chemicals which affect HPG axis (e.g. fipronil, muscimol) in fish (Ankley et al., 2009). As a result, we speculated that BT may have neurotoxicity, thus we analyzed the response of the brain proteome of rare minnow (Liang et al., unpublished data). In this experiment, a total of 41 differentially expressed proteins were identified and pathways including cellular respiration, G-protein signal cascades, $\mathrm{Ca}^{2+}$-dependent signaling, and cell cycle and apoptosis were affected by BTR. In addition, several proteins related to neurodegeneration were identified in this study (e.g. HPCA, 14-3-3 protein epsilon; YWHAE, glutamine synthetase 1; GS, Voltage-dependent anion channel; VDAC), which provides insight into the BT induced molecular responses in the brain that underlie neurotoxicity.

\section{Limitations and future}

Compared with other model fish, the genetic and protein database of rare minnows remain somewhat limited, although expressed sequence tags from the liver and some cDNA sequences have been generated (Wei et al., 2007). We searched NCBI for the nucleotide, protein and EST database of zebrafish (Danio rerio), medaka (Oryzias latipes), fathead minnow (Pimephales promelas), and rare minnow (Gobiocypris rarus). The amount of sequence information in rare minnows is far below that of other species (Fig. 4). As a result, limited protein information of rare minnow as well as their functional annotation remains a challenge. The aforementioned studies of liver proteomes of rare minnows exposed to EDCs (Fang et al., 2010; Liang et al., 2014a; Wei et al., 2008a) show that many altered proteins could not be identified, and only a small set of proteins were actually identified and interpreted.

To date, there are only a few quantitative proteomic-based studies performed in rare minnows, and all based on gel-based technologies. However, a serious limitation of proteomic analysis using 2D gel electrophoresis is the sensitivity of detection. Analysis of low abundance proteins by 2D electrophoresis is challenging due to the presence of high abundant proteins (Roe and Griffin, 2006). A typical 2D gel using the large format gel detect at most 1000 proteins, a low number if one 


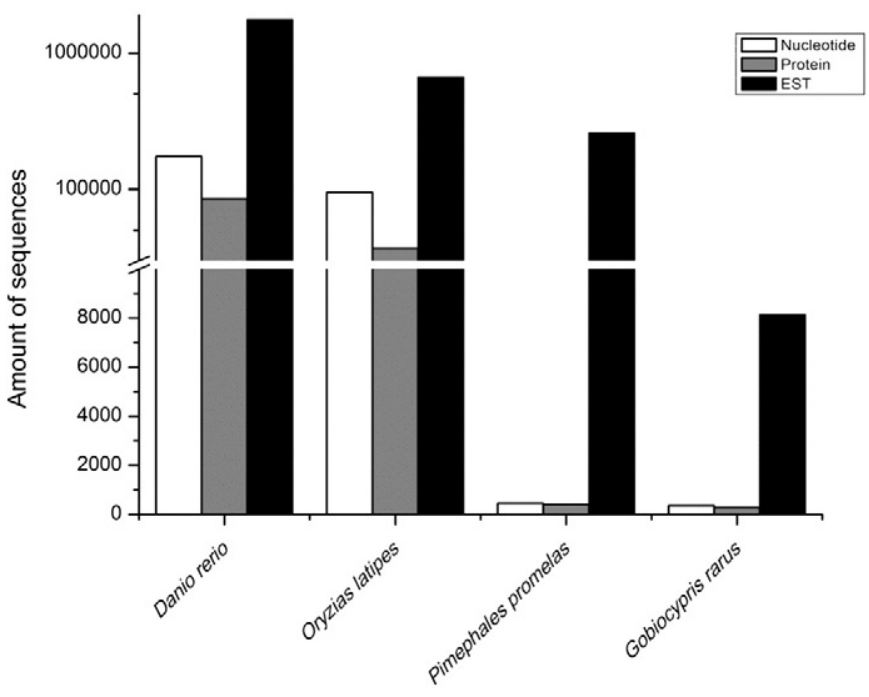

Fig. 4. Comparison of numbers of nucleotide (white bars), protein (grey bars) and EST sequences (black bars) for zebrafish (Danio rerio), medaka (Oryzias latipes), fathead minnow (Pimephales promelas), and rare minnow (Gobiocypris rarus).

considers that about 10,000-15,000 genes may be expressed in a tissue in addition to a number of isoforms and post-translated proteins. Nongel based methods can detect up 6000-7000 proteins and therefore cover more of the proteome (Baggerman et al., 2005). As a result, more and more researches have begun to use gel-free proteomics approaches in aquatic toxicological studies. For example, Ralston-Hooper et al. (2012) applied a comprehensive, liquid chromatography-tandem mass spectrometry (LC-MS/MS)-based proteomic approach to identify and quantify differentially expressed hepatic proteins from female fathead minnows exposed to fadrozole. They identified 782 proteins and demonstrate a gel-free, label-free method is feasible and effective to determine differentially expressed proteins in small fish species after toxicant exposure. Therefore, gel-free methods conducted in rare minnows in the future will increase peptide/protein identification and achieve more valuable information to improve our understanding in toxic mechanisms of chemicals in this species.

Another limitation in toxicogenomics studies in rare minnows has been that only one or very few concentrations were analyzed, and only at one point in time. Temporal patterns in gene expression may provide an important representation of the regulation that occurs in response to toxic effects in time. However, gene expression undergoes distinct temporal phases in response to toxicants (Ankley and Villeneuve, 2015). In addition, differential regulatory mechanisms complicate gene-protein relationships, but provide different information depending on the temporal scale. Thus, time-course studies are expected to provide new insights into gene-protein relationships in fish in future studies.

In summary, Chinese rare minnow (Gobiocypris rarus) is an excellent model for chemical safety assessment and environmental toxicological studies. With the development of toxicogenomic technologies, studies using rare minnows in response to environmental stress continue to become more efficient and informative despite its current challenges and limitations. As toxicology moves away from a single mechanism of action approach toward a toxicity pathway-based paradigm, toxicogenomic data from omics technologies are expected to be integrated to better understand the underlying physiology and how fish interact with their environment. Additionally, toxicogenomics offers great potential as a biomarker discovery tool as well as for increasing our understanding of the effects of mixtures. Thus, expanding toxicogenomics reference database and a better understanding of the relationships between specific responses and biomarkers to stress can also improve chemical risk assessment and environmental monitoring.

\section{Acknowledgments}

We acknowledge Professor Christopher J. Martyniuk (Center for Environmental and Human Toxicology \& Department of Physiological Sciences, University of Florida) for his scientific expertise in reviewing this article and English language editing. This review was supported by Natural Science Foundation of Inner Mongolia Autonomous Region of China (2015MS0202).

\section{References}

Afshari, C.A., Nuwaysir, E.F., Barrett, J.C., 1999. Application of complementary DNA microarray technology to carcinogen identification, toxicology, and drug safety evaluation. Cancer Res. 59, 4759-4760.

Ahmad, Y., Lamond, A.I., 2014. A perspective on proteomics in cell biology. Trends Cell Biol. 24, 257-264.

Altenburger, R., Scholz, S., Schmitt-Jansen, M., Busch, W., Escher, B.I., 2012. Mixture toxicity revisited from a toxicogenomic perspective. Environ. Sci. Technol. 46, 2508-2522.

Ankley, G.T., Villeneuve, D.L., 2015. Temporal changes in biological responses and uncertainty in assessing risks of endocrine-disrupting chemicals: insights from intensive time-course studies with fish. Toxicol. Sci. 259-275.

Ankley, G.T., Daston, G.P., Degitz, S.J., Denslow, N.D., Hoke, R.A., Kennedy, S.W., Miracle, A.L., Perkins, E.J., Snape, J., Tillitt, D.E., 2006. Toxicogenomics in regulatory ecotoxicology. Environ. Sci. Technol. 40, 4055-4065.

Ankley, G.T., Bencic, D.C., Breen, M.S., Collette, T.W., Conolly, R.B., Denslow, N.D., Edwards, S.W., Ekman, D.R., Garcia-Reyero, N., Jensen, K.M., 2009. Endocrine disrupting chemicals in fish: developing exposure indicators and predictive models of effects based on mechanism of action. Aquat. Toxicol. 92, 168-178.

Baggerman, G., Vierstraete, E., De Loof, A., Schoofs, L., 2005. Gel-based versus gel-free proteomics: a review. Comb. Chem. High Throughput Screen. 8, 669-677.

Boverhof, D.R., Zacharewski, T.R., 2006. Toxicogenomics in risk assessment: applications and needs. Toxicol. Sci. 89, 352-360.

Brown, P.O., Botstein, D., 1999. Exploring the new world of the genome with DNA microarrays. Nat. Genet. 21, 33-37.

Calzolai, L., Ansorge, W., Calabrese, E., Denslow, N., Part, P., Lettieri, T., 2007. Transcriptomics and proteomics. Applications to Ecotoxicology. Comp. Biochem. Physiol. Part D: Genomics and Proteomics 2, pp. 245-249.

Chang, J., Wang, J., Cao, W., 1995. The embryonic development of Gobiocypris rarus. Acta Hydrobiol. Sin. 2, 97-103.

de Sousa Abreu, R., Penalva, L.O., Marcotte, E.M., Vogel, C., 2009. Global signatures of protein and mRNA expression levels. Mol. BioSyst. 5, 1512-1526.

Dowling, V.A., Sheehan, D., 2006. Proteomics as a route to identification of toxicity targets in environmental toxicology. Proteomics 6, 5597-5604

Duggan, D.J., Bittner, M., Chen, Y., Meltzer, P., Trent, J., 1999. Expression profiling using cDNA microarrays. Nat. Genet. 21, 10-14.

Fang, Y., Gao, X., Zha, J., Ning, B., Li, X., Gao, Z., Chao, F., 2010. Identification of differential hepatic proteins in rare minnow (Gobiocypris rarus) exposed to pentachlorophenol (PCP) by proteomic analysis. Toxicol. Lett. 199, 69-79.

Fang, Y., Gao, X., Zhao, F., Zhang, H., Zhang, W., Yang, H., Lin, B., Xi, Z., 2014. Comparative proteomic analysis of ovary for Chinese rare minnow (Gobiocypris rarus) exposed to chlorophenol chemicals. J. Proteome 110, 172-182.

Forné, I., Abián, J., Cerdà, J., 2010. Fish proteome analysis: model organisms and nonsequenced species. Proteomics 10, 858-872.

Garcia-Reyero, N., Perkins, E.J., 2011. Systems biology: leading the revolution in ecotoxicology. Environ. Toxicol. Chem. 30, 265-273.

Gatzidou, E.T., Zira, A.N., Theocharis, S.E., 2007. Toxicogenomics: a pivotal piece in the puzzle of toxicological research. J. Appl. Toxicol. 27, 302-309.

General Administration of Quality Supervision Inspection and Quarantine of the People's Republic of China, Standardization Administration of the People's Republic of China, 2014n. Chemicals-rare Minnow (Gobiocypris rarus) Acute Toxicity Test. China Standards Press, Beijing, China.

Hawliczek, A., Nota, B., Cenijn, P., Kamstra, J., Pieterse, B., Winter, R., Winkens, K., Hollert, H., Segner, H., Legler, J., 2012. Developmental toxicity and endocrine disrupting potency of 4-azapyrene, benzo[b]fluorene and retene in the zebrafish Danio rerio. Reprod. Toxicol. 33, 213-223.

Hontela, A., 1998. Interrenal dysfunction in fish from contaminated sites: in vivo and in vitro assessment. Environ. Toxicol. Chem. 17, 44-48.

Ju, Z., Dunham, R.A., Liu, Z., 2002. Differential gene expression in the brain of channel catfish (Ictalurus punctatus) in response to cold acclimation. Mol. Gen. Genomics. 268, 87-95.

Ju, Z., Wells, M.C., Walter, R.B., 2007. DNA microarray technology in toxicogenomics of aquatic models: methods and applications. Comp. Biochem. Physiol. C: Toxicol. Pharmacol. 145, 5-14.

Kosmehl, T., Otte, J.C., Yang, L., Legradi, J., Bluhm, K., Zinsmeister, C., Keiter, S.H., Reifferscheid, G., Manz, W., Braunbeck, T., Strähle, U., Hollert, H., 2012. A combined DNA-microarray and mechanism-specific toxicity approach with zebrafish embryos to investigate the pollution of river sediments. Reprod. Toxicol. 33, 245-253.

Lankadurai, B.P., Nagato, E.G., Simpson, M.J., 2013. Environmental metabolomics: an emerging approach to study organism responses to environmental stressors. Environ. Rev. 21, 180-205.

Larkin, P., Folmar, L.C., Hemmer, M.J., Poston, A.J., Lee, H.S., Denslow, N.D., 2002a. Array technology as a tool to monitor exposure of fish to xenoestrogens. Mar. Environ. Res. 54, 395-399. 
Larkin, P., Sabo-Attwood, T., Kelso, J., Denslow, N.D., 2002b. Gene expression analysis of largemouth bass exposed to estradiol, nonylphenol, and p, p'-DDE. Comp. Biochem. Physiol. B Biochem. Mol. Biol. 133, 543-557.

Lettieri, T., 2006. Recent applications of DNA microarray technology to toxicology and ecotoxicology. Environ. Health Perspect. 114, 4-9.

Li, W., Zha, J., Li, Z., Yang, L., Wang, Z., 2009a. Effects of exposure to acetochlor on the expression of thyroid hormone related genes in larval and adult rare minnow (Gobiocypris rarus). Aquat. Toxicol. 94, 87-93.

Li, W., Zha, J., Spear, P.A., Li, Z., Yang, L., Wang, Z., 2009b. Changes of thyroid hormone levels and related gene expression in Chinese rare minnow (Gobiocypris rarus) during 3-amino-1, 2, 4-triazole exposure and recovery. Aquat. Toxicol. 92, 50-57.

Li, M., Wang, J., Lu, Z., Wei, D., Yang, M., Kong, L., 2014. NMR-based metabolomics approach to study the toxicity of lambda-cyhalothrin to goldfish (Carassius auratus). Aquat. Toxicol. 146, 82-92.

Liang, X., Martyniuk, C.J., Cheng, G., Zha, J., Wang, Z., 2014a. Pyruvate carboxylase as a sensitive protein biomarker for exogenous steroid chemicals. Environ. Pollut. 189, 184-193.

Liang, X., Wang, M., Chen, X., Zha, J., Chen, H., Zhu, L., Wang, Z., 2014b. Endocrine disrupting effects of benzotriazole in rare minnow (Gobiocypris rarus) in a sexdependent manner. Chemosphere 112, 154-162.

Martyniuk, C.J., Denslow, N.D., 2009. Towards functional genomics in fish using quantitative proteomics. Gen. Comp. Endocrinol. 164, 135-141.

Martyniuk, C.J., Denslow, N.D., 2012. Exploring androgen-regulated pathways in teleost fish using transcriptomics and proteomics. Integr. Comp. Biol. 52, 695-704.

Martyniuk, C.J., Kroll, K.J., Doperalski, N.J., Barber, D.S., Denslow, N.D., 2010a. Environmentally relevant exposure to $17 \alpha$-ethinylestradiol affects the telencephalic proteome of male fathead minnows. Aquat. Toxicol. 98, 344-353.

Martyniuk, C.J., Kroll, K.J., Doperalski, N.J., Barber, D.S., Denslow, N.D., 2010b. Genomic and proteomic responses to environmentally relevant exposures to dieldrin: indicators of neurodegeneration? Toxicol. Sci. 117, 190-199.

Martyniuk, C.J., Alvarez, S., Denslow, N.D., 2012a. DIGE and iTRAQ as biomarker discovery tools in aquatic toxicology. Ecotoxicol. Environ. Saf. 76, 3-10.

Martyniuk, C.J., Popesku, J.T., Chown, B., Denslow, N.D., Trudeau, V.L., 2012b. Quantitative proteomics in teleost fish: insights and challenges for neuroendocrine and neurotoxicology research. Gen. Comp. Endocrinol. 176, 314-320.

Miracle, A.L., Ankley, G.T., 2005. Ecotoxicogenomics: linkages between exposure and effects in assessing risks of aquatic contaminants to fish. Reprod. Toxicol. 19, 321-326.

Mitra, P.S., Ghosh, S., Zang, S., Sonneborn, D., Hertz-Picciotto, I., Trnovec, T., Palkovicova, L. Sovcikova, E., Ghimbovschi, S., Hoffman, E.P., Dutta, S.K., 2012. Analysis of the toxicogenomic effects of exposure to persistent organic pollutants (POPs) in Slovakian girls: correlations between gene expression and disease risk. Environ. Int. 39, 188-199.

Nelson, J.S., 1994. Fishes of the World. John Wiley \& Sons, New York.

Nesatyy, V.J., Suter, M.J.F., 2007. Proteomics for the analysis of environmental stress responses in organisms. Environ. Sci. Technol. 41, 6891-6900.

Nuwaysir, E.F., Bittner, M., Trent, J., Barrett, J.C., Afshari, C.A., 1999. Microarrays and toxicology: the advent of toxicogenomics. Mol. Carcinog. 24, 153-159.

Oberemm, A., Onyon, L., Gundert-Remy, U., 2005. How can toxicogenomics inform risk assessment? Toxicol. Appl. Pharmacol. 207, 592-598.

Patel, V.J., Thalassinos, K., Slade, S.E., Connolly, J.B., Crombie, A., Murrell, J.C., Scrivens, J.H., 2009. A comparison of labeling and label-free mass spectrometry-based proteomics approaches. J. Proteome Res. 8, 3752-3759.

Popesku, J.T., Martyniuk, C.J., Denslow, N.D., Trudeau, V.L., 2010. Rapid dopaminergic modulation of the fish hypothalamic transcriptome and proteome. PLoS One 5, e12338.

Poynton, H.C., Vulpe, C.D., 2009. Ecotoxicogenomics: emerging technologies for emerging contaminants1. J. Am. Water Resour. Assoc. 45, 83-96.

Qun-Fang, Z., Gui-Bin, J., Ji-Yan, L., 2002. Effects of sublethal levels of tributyltin chloride in a new toxicity test organism: the Chinese rare minnow (Gobiocypris rarus). Arch. Environ. Contam. Toxicol. 42, 332-337.

Ralston-Hooper, K., Turner, M., Soderblom, E., Villeneuve, D., Ankley, G., Moseley, M., Hoke, R., Ferguson, P., 2012. Application of a label-free, gel-free quantitative proteomics method for ecotoxicological studies of small fish species. Environ. Sci. Technol. 47, 1091-1100.

Roe, M.R., Griffin, T.J., 2006. Gel-free mass spectrometry-based high throughput proteomics: tools for studying biological response of proteins and proteomes. Proteomics 6, 4678-4687.

Sanchez, B.C., Ralston-Hooper, K., Sepúlveda, M.S., 2011. Review of recent proteomic applications in aquatic toxicology. Environ. Toxicol. Chem. 30, 274-282.

Schmidt, C.W., 2002. Toxicogenomics: an emerging discipline. Environ. Health Perspect. 110, A750-A755.
Shepard, J., Bradley, B., 2000. Protein expression signatures and lysosomal stability in Mytilus edulis exposed to graded copper concentrations. Mar. Environ. Res. 50 457-463.

Shepard, J., Olsson, B., Tedengren, M., Bradley, B., 2000. Protein expression signatures identified in Mytilus edulis exposed to PCBs, copper and salinity stress. Mar. Environ. Res. 50, 337-340.

Tuffnail, W., Mills, G., Cary, P., Greenwood, R., 2009. An environmental ${ }^{1} \mathrm{H}$ NMR metabolomic study of the exposure of the marine mussel Mytilus edulis to atrazine, lindane, hypoxia and starvation. Metabolomics 5, 33-43.

Viant, M.R., Sommer, U., 2013. Mass spectrometry based environmental metabolomics: a primer and review. Metabolomics 9, 144-158.

Wang, J., 1992. Reproductive biology of Gobiocypris rarus. Acta Hydrobiol. Sin. 16 165-174.

Wang, J., Cao, W., 1997. Gobiocypris rarus and fishes as laboratory animals. Trans. Chin. Ichthyol. Soc. 6, 144-152.

Wang, J., Song, T., Cao, W., 1998. Postembryonic development and growth of cultured rare minnow, Gobiocypris rarus. Acta Hydrobiol. Sin. 2, 128-134.

Waters, M.D., Fostel, J.M., 2004. Toxicogenomics and systems toxicology: aims and prospects. Nat. Rev. Genet. 5, 936-948.

Wei, Y., Wang, J., Zhang, X., Xu, M., Dai, J., 2007. Identification and characterization of expressed sequence tags from the liver of rare minnow (Gobiocypris rarus). Comp. Biochem. Physiol. Part D Genomics Proteomics 2, 356-362.

Wei, Y., Chan, L.L., Wang, D., Zhang, H., Wang, J., Dai, J., 2008a. Proteomic analysis of hepatic protein profiles in rare minnow (Gobiocypris rarus) exposed to perfluorooctanoic acid. J. Proteome Res. 7, 1729-1739.

Wei, Y., Liu, Y., Wang, J., Tao, Y., Dai, J., 2008b. Toxicogenomic analysis of the hepatic effects of perfluorooctanoic acid on rare minnows (Gobiocypris rarus). Toxicol. Appl. Pharmacol. 226, 285-297.

Wei, Y., Shi, X., Zhang, H., Wang, J., Zhou, B., Dai, J., 2009. Combined effects of polyfluorinated and perfluorinated compounds on primary cultured hepatocytes from rare minnow (Gobiocypris rarus) using toxicogenomic analysis. Aquat. Toxicol 95, 27-36.

Wetmore, B.A., Merrick, B.A., 2004. Invited review: toxicoproteomics: proteomics applied to toxicology and pathology. Toxicol. Pathol. 32, 619-642.

Yang, L., Zha, J., Li, W., Li, Z., Wang, Z., 2010a. Atrazine affects kidney and adrenal hormones (AHs) related genes expressions of rare minnow (Gobiocypris rarus). Aquat. Toxicol. 97, 204-211.

Yang, L., Zha, J., Zhang, X., Li, W., Li, Z., Wang, Z., 2010b. Alterations in mRNA expression of steroid receptors and heat shock proteins in the liver of rare minnow (Grobiocypris rarus) exposed to atrazine and p, p'-DDE. Aquat. Toxicol. 98, 381-387.

Zha, J., Wang, Z., Wang, N., Ingersoll, C., 2007. Histological alternation and vitellogenin induction in adult rare minnow (Gobiocypris rarus) after exposure to ethynylestradio and nonylphenol. Chemosphere 66, 488-495.

Zha, J., Sun, L., Zhou, Y., Spear, P.A., Ma, M., Wang, Z., 2008. Assessment of $17 \alpha-$ ethinylestradiol effects and underlying mechanisms in a continuous, multigeneration exposure of the Chinese rare minnow (Gobiocypris rarus). Toxicol. Appl. Pharmacol. 226, 298-308.

Zhang, X., Yang, F., Zhang, X., Xu, Y., Liao, T., Song, S., Wang, J., 2008a. Induction of hepatic enzymes and oxidative stress in Chinese rare minnow (Gobiocypris rarus) exposed to waterborne hexabromocyclododecane (HBCDD). Aquat. Toxicol. 86, 4-11.

Zhang, X., Zha, J., Li, W., Yang, L., Wang, Z., 2008b. Effects of 2, 4-dichlorophenol on the expression of vitellogenin and estrogen receptor genes and physiology impairments in Chinese rare minnow (Gobiocypris rarus). Environ. Toxicol. 23, 694-701.

Zhang, X., Zha, J., Wang, Z., 2008c. Influences of 4-nonylphenol on doublesex-and mab-3related transcription factor 1 gene expression and vitellogenin mRNA induction of adult rare minnow (Gobiocypris rarus). Environ. Toxicol. Chem. 27, 196-205.

Zhang, L., Liu, X., You, L, Zhou, D., Wu, H. Li, L, Zhao, J., Feng, J., Yu, J., 2011. Metabolic responses in gills of Manila clam Ruditapes philippinarum exposed to copper using NMR-based metabolomics. Mar. Environ. Res. 72, 33-39.

Zhang, Y., Fonslow, B.R., Shan, B., Baek, M.-C., Yates III, J.R., 2013. Protein analysis by shotgun/bottom-up proteomics. Chem. Rev. 113, 2343-2394.

Zhong, X., Xu, Y., Liang, Y., Liao, T., Wang, J., 2005. The Chinese rare minnow (Gobiocypris rarus) as an in vivo model for endocrine disruption in freshwater teleosts: a full lifecycle test with diethylstilbestrol. Aquat. Toxicol. 71, 85-95.

Zhou, Y., Cheng, S., Hu, W., Sun, M., 1995. A new toxicity test organism-Gobiocypris rarus. Zool. Res. 16, 59-63. 